Carnets de géographes

GÉOGRAPHES

\title{
Le système des villes moyennes du sud du chili, vers la construction de nouveaux espaces de relations?
}

\section{Francisco Maturana}

\section{(2) OpenEdition}

Journals

Édition électronique

URL : http://journals.openedition.org/cdg/1109

DOI : $10.4000 /$ cdg. 1109

ISSN : 2107-7266

Éditeur

UMR 245 - CESSMA

Référence électronique

Francisco Maturana, «Le système des villes moyennes du sud du chili, vers la construction de nouveaux espaces de relations? », Carnets de géographes [En ligne], 5 | 2013, mis en ligne le 01 janvier 2013, consulté le 24 septembre 2020. URL : http://journals.openedition.org/cdg/1109 ; DOI : https:// doi.org/10.4000/cdg. 1109

\section{cc) $($ () $\ominus$}

La revue Carnets de géographes est mise à disposition selon les termes de la Licence Creative Commons Attribution - Pas d'Utilisation Commerciale - Pas de Modification 4.0 International. 


\section{LE SYSTÈME DES VILLES MOYENNES DU SUD DU CHILI, VERS LA CONSTRUCTION DE NOUVEAUX ESPACES DE RELATIONS?}

FRANCISCO MATURANA

La question des villes moyennes ou intermédiaires a été largement débattue, en raison de la difficulté à établir leur définition. Les différentes recherches sur les villes moyennes et intermédiaires montrent que le débat sur les concepts est très ouvert et très développé en géographie. Les appréciations des concepts varient selon les pays et selon le rôle que possède chaque ville moyenne à l'intérieur de l'armature urbaine.

Il est possible en reliant le concept de ville intermédiaire aux "fonctions territoriales ", que nous pourrions définir comme : l'ensemble des activités d'une ville qui ont un impact sur l'organisation spatiale du système, ainsi que sur le flux des personnes et des marchandises. II s'agirait d'interactions entre différentes villes (ou territoires) qui provoqueraient des liens de dépendance ou de concurrence, ce qui se traduit par une articulation particulière de la hiérarchie urbaine, selon l'impact occasionné par la fonction spatiale de la ville et les rapports entre les villes.

Un système particulier de villes moyennes se trouve situé au sud du Chili, qui est constitué par les régions La Araucanía, Los Ríos et Los Lagos (voir figure 1). Du point de vue de l'organisation spatiale, même si le paradigme de la place centrale semble dominer, certaines caractéristiques du paradigme du réseau sont visibles, spécialement là où la taille démographique d'une ville ne détermine pas la dynamique et l'influence hiérarchique que peut avoir le centre urbain dans son territoire. De toute façon, bien que les villes de cette région ne présentent pas de dépendance capitale envers un centre urbain de plus grande taille, leur autonomie, en termes de services ou d'infrastructure, est encore peu développée. En effet, ce système présente une configuration spatiale plutôt monocentrique, où les interactions entre villes se développent à l'intérieur de chaque région, et les liens transfrontaliers sont faibles voire inexistants. 
Figure 1 : Le système des villes moyennes du sud du Chili

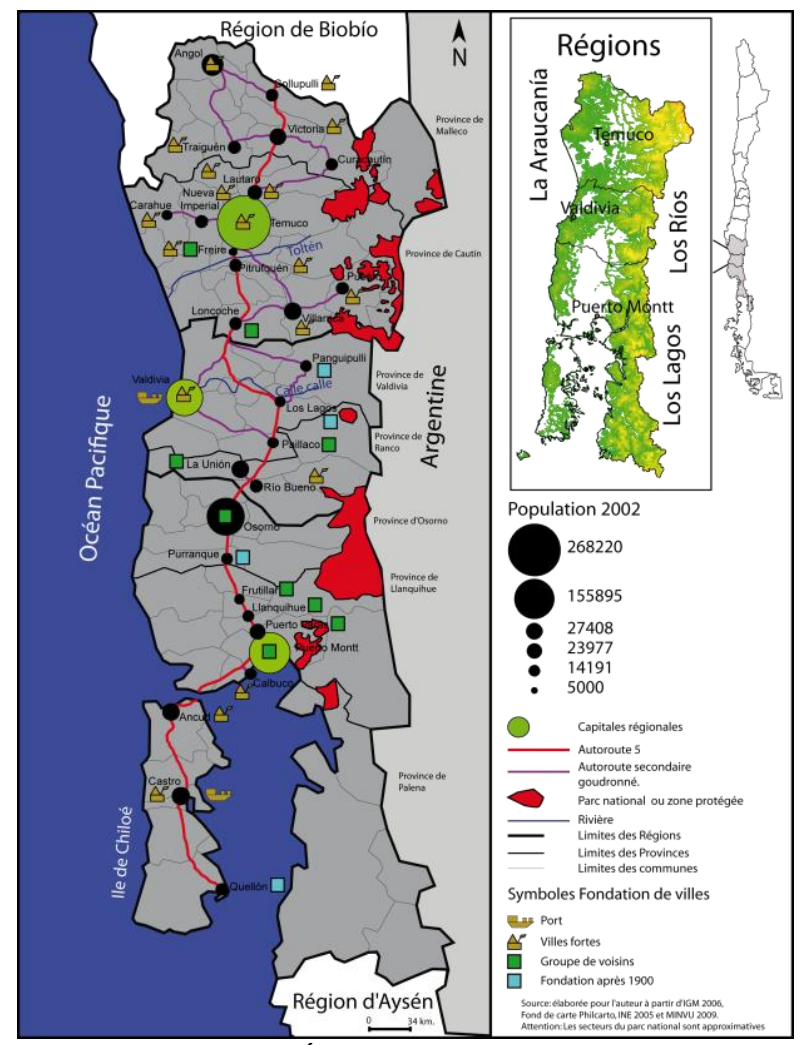

Source : Élaboration personnelle

Les centres urbains du sud du Chili à l'intérieur de chaque région entretiennent trois types de relations (voir figure 2). Le premier type, que nous avons nommé de forte domination, possède une dominance par sa taille et par la présence d'aspects fonctionnels. En effet, il s'agit de deux centres urbains où l'un d'entre eux est de plus grande taille que l'autre. La dominance du centre de plus grande taille est liée aux aspects économiques et administratifs, la concentration de biens et de services, d'infrastructure, d'éducation et d'emploi sont des caractéristiques de cette domination. La proximité ne serait pas déterminante dans cette configuration, car le centre dominant de grande taille pourrait gérer un rayon de domination presque à échelle régionale sur un autre centre urbain. Des exemples en sont la ville de Temuco et sa dominance sur les centres urbains de Nueva Imperial ou Lautaro, mais c'est aussi le cas de Puerto Montt et son effet sur la ville de Llanquihue, ou, sur des distances géographiques plus éloignées, l'influence de la ville de Temuco sur Victoria.

Dans le deuxième type (domination moyenne), il s'agit de deux centres urbains de tailles similaires ou parfois différentes (sans présenter une grande disparité), où l'un d'eux a une dominance sur l'autre, du fait de leur proximité géographique, d'une activité économique ou d'aspects fonctionnels. C'est le cas des villes de La Unión et Río Bueno ou de Castro et Ancud.

Dans un troisième type, nous trouvons une "relation d'équivalence " où il est possible d'observer des villes de taille similaire ou non, géographiquement proches ou non, mais qui entretiennent un grand nombre de flux égaux sans présenter de dominance entre elles. Généralement, ces deux centres sont dominés par un troisième nœud supérieur dans la hiérarchie, des exemples en sont Paillaco et Río Bueno, Victoria et Curacautín. 
Figure 2 : Les types de relations des villes du sud du Chili

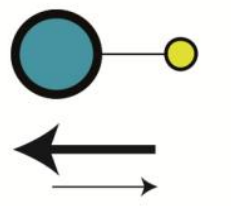

Forte domination

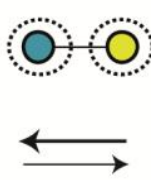

Moyenne domination

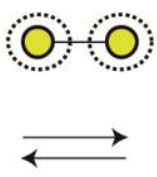

Relation d'équivalence

Source : Élaboration personnelle

À l'échelle régionale, nous pouvons constater trois types de configurations (voir figure 3), la première organisation est de type étoile, elle est constituée de plusieurs villes dominées par un grand centre urbain. II s'agit d'un réseau monocentrique à forte relation hiérarchique verticale. Les flux se dirigent plutôt vers le centre et dans ce cas l'attraction est liée à l'emploi, l'éducation, à l'infrastructure et aux services. Par la disposition géographique des villes dans l'espace nous pourrions dire que cette organisation est présente dans la région de La Araucanía.

Figure 3 : Trois types de configurations dans l'espace régionale au Chili
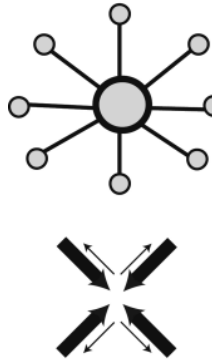

Organisation type étoile
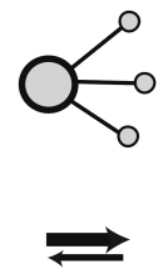

Organisation type connecteur noeud linéaire
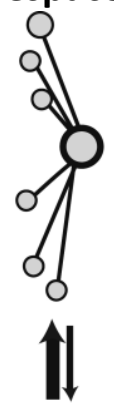

Organisation type noeud Organisation type noe elle

Le deuxième type d'organisation est du type connecteur de nœuds linéaire, qui présente une dépendance moyenne entre villes dans l'ensemble urbain et qui montre, en dépit de l'existence d'un grand centre urbain et son rôle concentrateur, que d'autres nœuds du réseau ont une certaine autonomie de relations réciproques sans la capitale régionale. Les flux sont établis dans un sens est-ouest et vice versa (géographiquement). II s'agit notamment de l'organisation des villes qui se développent dans la région de Los Ríos.

La troisième configuration possède un grand centre à forte hiérarchie. Les relations entre villes se font dans un sens géographique, plutôt situé nord-sud et vice versa, avec une caractéristique de connexion linéaire où la communication entre les nœuds est un grand axe qui les connecte tous de façon continue. Fonctionnellement, les relations entre centres urbains se font plutôt de façon verticale et hiérarchique. Par ailleurs, il existe un deuxième nœud d'importante taille, mais dont l'influence est limitée. À l'intérieur de l'organisation, des nœuds s'organisent certains territoires fermés ou isolés. II s'agit de la région de Los Lagos. 
Fiche informative

\section{Lien électronique}

http://geografia.udec.cl/fmaturana/These MATURANA/

Discipline

Géographie

Directeur

ROBERT, Jean

\section{Université}

Université Paris-Sorbonne Paris IV

Membres du jury de thèse, soutenue le 12 mars 2012

- M. ARENAS, Federico Professeur à I'Université Pontificale Catholique du Chili

- MME BRETAGNOLLE Professeure à I'Université Paris 1-Panthéon-Sorbonne

- M. CARRIERE Professeur émérite à I'Université de Tours

- M. ROBERT Professeur à I'Université Paris-Sorbonne Paris IV

\section{Situation professionnelle actuelle}

Assistant professor à Universidad de Concepción, Chile

\section{Courriel de l'auteur}

mailto:franmaturana@udec.cl 